\title{
A New Approach to 3D Sulcal Ribbon Finding from MR Images
}

\author{
X.Zeng ${ }^{1}$, L.H.Staib ${ }^{1}$, R.T.Schultz ${ }^{2}$, H.Tagare ${ }^{1}$, L.Win ${ }^{2}$, and J.S.Duncan ${ }^{1}$ \\ 1 Departments of Electrical Engineering and Diagnostic Radiology \\ ${ }^{2}$ Child Study Center \\ Yale University, New Haven, CT, 06520-8042
}

\begin{abstract}
Sulcal medial surfaces are 3D thin convoluted ribbons embedded in cortical sulci, and they provide distinctive anatomical features of the brain. Here we propose a new approach to automatic intrasulcal ribbon finding, following our work on cortex segmentation with coupled surfaces via level set methods, where the outer cortical surface is embedded as the zero level set of a high-dimensional distance function. Through the utilization of this distance function, we are able to formulate the sulcal ribbon finding problem as one of surface deformation, thus avoiding possible control problems in other work using sliding contour models. Using dynamic programming and deformable surface models, our method requires little manual intervention and results parameterized sulcal ribbon surfaces in nearly real-time. Though a natural follow up to our earlier segmentation work, we describe how it can be applied with general segmentation methods. We also present quantitative results on $15 \mathrm{MR}$ brain images.
\end{abstract}

\section{Introduction}

A great amount of recent anatomical MRI studies of the human brain have been focused on the cerebral cortex, which is characterized by its convoluted cortical surface. The narrow groove separating adjacent cortical convolutions is called a sulcus, and the intrasulcal medial surface can be modeled as a 3D thin ribbon embedded in the cortical fold. The deepest part of a sulcus is called the fundus, and it often demarcates the boundary between cortical regions with observable differences in their cytoarchitecture (the packing density and laminar distribution of different neuron types) and function [14].

Because of the importance of sulcal ribbons in brain structural and functional analysis, a number of recent efforts have begun to deal with the automatic extraction of sulci $[15,8]$, the probabilistic study of sulcal geometry and configuration [9], and automatic sulcal labeling [11].

Of all the work cited above, ours is most closely related to that of Vaillant and Davatzikos [15]. The aim is to automatically extract a sulcal ribbon surface and provide a parametric representation, thereby further facilitating quantitative shape analysis and cortical-constrained brain matching and warping. Vaillant and Davatzikos start by initializing an active contour at the exterior part of a 
sulcus (see Figure 1). A parametric representation of the sulcal medial surface is obtained as the active contour slide down toward the deep sulcal bottom under the influence of weighted external forces, such as the center-of-mass force and the inward force which is a combination based on surface normal, curve sliding speed and sliding acceleration. This deformable model uses characteristics of the cortical shape, and has been successfully applied to MR brain images to extract sulcal ribbons. However, the manual placement of the initializing curve is a limitation, and so could be the tuning of the weights on the external forces.

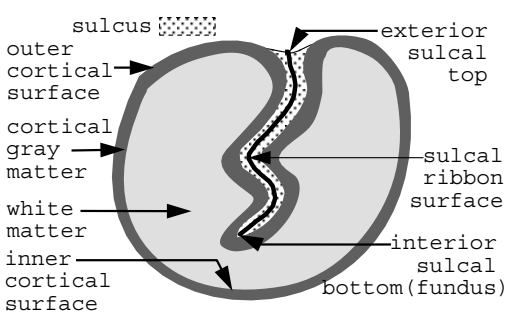

(a)

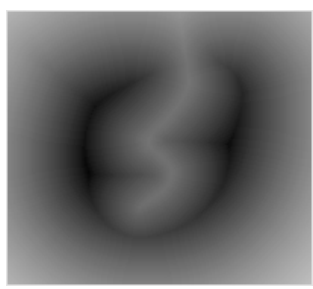

(b)

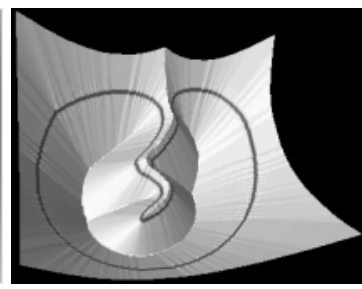

(c)

Fig. 1. (a): 2D schematic representation of a sulcus; Gray scale view (b) and surface view (c) of the signed distance function corresponding to the outer cortical boundary shown in (a).

\section{Our Method}

Following our earlier work on cortex segmentation with coupled surfaces using a level set implementation [19], we propose a new approach to automatic sulcal ribbon finding. Through the utilization of the distance function in which the outer cortical surface is embedded as its zero level set, we are able to formulate the sulcal ribbon finding problem as one of surface deformation, avoiding possible control problems of tuning weights on external forces in the sliding contour method. Our sulcal ribbon finding algorithm starts from the outer cortical surface and its associated level function, and takes three steps as shown in Figure 2.

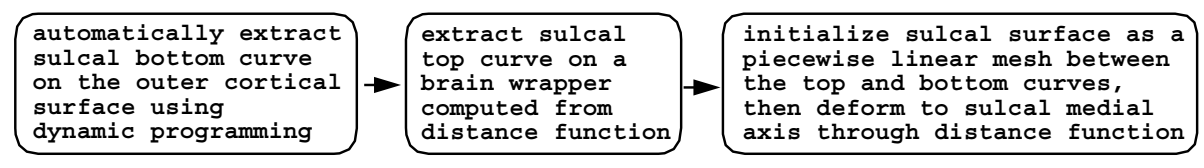

Fig. 2. Diagram of our algorithm.

\subsection{Brain Segmentation}

We first perform segmentation of brain images using our coupled surfaces propagation algorithm with a level set implementation [19]. Starting from concentric spheres, the outer and inner surfaces propagate out, looking for image features 
of CSF/gray boundary (outer cortical surface) and gray/white boundary (inner cortical surface) respectively, while maintaining a nearly constant thickness in between. When the propagation ends, we have two level functions $\Psi_{\text {out }}$ and $\Psi_{\text {in }}$ in which the outer and inner cortical surfaces $S_{\text {out }}$ and $S_{\text {in }}$ are embedded as zero level sets respectively.

Because of the level set implementation, our segmentation algorithm has the advantage of handling highly convoluted structures. As a result, $S_{\text {out }}$ captures the deep sulcal folds and gets down to the interior sulcal bottom (see Figure 1 and Figure 5) rather than staying at the exterior sulcal top. Therefore, we can use this surface to extract a sulcal bottom curve at the fundus, which greatly facilitates the sulcal ribbon finding. This is an important difference of our sulcal ribbon finding from that due to Vallaint and Davatzikos.

Though the sulcal ribbon finding algorithm proposed here is a natural follow up to our earlier segmentation work, it easily applies in general settings. $S_{\text {out }}$ can be extracted from any segmented brain volume using iso-surface based methods with specific constraints to preserve high curvature areas, such as that proposed by Gibson [7]. $\Psi_{\text {out }}$ can then be calculated as the signed distance function:

$$
\Psi_{\text {out }}(x)=-\operatorname{dist}\left(x, S_{\text {out }}\right) \quad \text { if } x \in \text { brainvolume, }
$$

where dist is the Euclidean distance from position $x$ on $3 \mathrm{D}$ image grid to $S_{\text {out }}$. Narrow band techniques [20] can be used here to limit the calculation of $\Psi_{\text {out }}$ to positions close to $S_{\text {out }}$ for computational efficiency.

\subsection{Automatic Tracing of Sulcal Curves on Outer Cortical Surface}

Our first step in sulcal ribbon finding is to define the interior sulcal bottom curve at the fundus. Based on the fact that crest curves consist of points corresponding to local maxima of maximum principal curvature, defining sulcal bottom curves becomes a problem of finding curves that pass through regions of high maximum principal curvature while confined to the outer cortical surface. In the continuous case, this can be posed as a problem of finding curvature-weighted minimal geodesic curves. On our discretized outer cortical surface net, the goal is to find paths that go through surface vertices corresponding to maximal curvature values. The maximum principal curvature on each vertex $C u r v\left(v_{i}\right)$ on $S_{\text {out }}$ is calculated directly from $\Psi_{\text {out }}$ as described in [19]. Figure 3 shows an example of high maximum principal curvature points on an outer cortical surface.

We introduce the following notation for the description of our automatic curve extraction method.

- $V=\left\{v_{i} \mid 0 \leq i<M\right\}$ : the set of all vertices on the surface net, where $M$ is the number of vertices;

- $T=\left\{t_{j}\right\}$ : the set of all triangles on the surface net;

- $E=\left\{e_{i, j}\right\}$ : the set of all edges on the surface net, where $e_{i, j}$ is an edge if for some triangle $t \in T, v_{i}, v_{j}$ are vertices of $t . e_{i, j}$ is a degenerate edge if $i=j$.

- $N\left(v_{i}\right)=\left\{v_{j} \mid e_{i, j} \in E\right\}$ : the set of neighbors of vertex $v_{i}$; 
- $\operatorname{Cost}\left(e_{i, j}\right)=\operatorname{cost}\left(\operatorname{Curv}\left(v_{i}\right), \operatorname{Curv}\left(v_{j}\right)\right) \cdot \operatorname{dist}\left(v_{i}, v_{j}\right)$ : the cost of stepping through edge $e_{i, j}$, where function $\operatorname{cost}($,$) penalizes small maximum principal curvatures,$ and $\operatorname{dist}\left(v_{i}, v_{j}\right)$ gives the Euclidean distance between vertices $v_{i}$ and $v_{j}$. Function $\operatorname{cost}\left(\operatorname{Curv}\left(v_{i}\right), \operatorname{Curv}\left(v_{j}\right)\right)$ can take on forms such as $\operatorname{Curv} v_{\max }-\frac{\operatorname{Curv}\left(v_{i}\right)+\operatorname{Curv}\left(v_{j}\right)}{2}$, where $C u r v_{\max }$ is the largest maximum principal curvature of all surface vertices.

- $P_{i_{0}, i_{1}, \ldots, i_{K}}$ : a path from $v_{i_{0}}$ to $v_{i_{K}}$, consisting of a sequence of edges $e_{i_{0}, i_{1}}, e_{i_{1}, i_{2}}$, $\ldots, e_{i_{K-1}, i_{K}}$ (degenerate edges are allowed), where $K$ is the number of steps.

We now formulate the problem of finding a sulcal curve given the starting point $v_{\text {start }}$ and the ending point $v_{\text {end }}$, to be finding the optimal path:

$$
P^{*}=\arg \min _{P_{i_{0}, i_{2}, \ldots, i_{K}}} \Sigma_{k=0}^{K-1} \operatorname{Cost}\left(e_{i_{k}, i_{k+1}}\right)
$$

where $v_{i_{0}}=v_{\text {start }}, v_{i_{K}}=v_{\text {end }}$, and $K$ is the number of steps taken.

Dynamic programming $([2,3])$ is a technique suited for such an optimization problem. Similar application can be found in [10]. The basic idea is as follows. Suppose there are $M$ vertices on the surface net, then the optimal path $P^{*}$ takes no more than $M$ steps, i.e. $K \leq M$. If we can find an optimal path of step $K-1$ to the neighbors of $v_{e n d}$, then $P^{*}$ is just the optimal path to one particular neighbor of $v_{\text {end }}$, plus the edge from that particular neighbor to $v_{\text {end }}$.

The algorithm works in the following fashion. Initialize a pathvalue for $v_{\text {end }}$ to be 0 , and $+\infty$ for the rest of the vertices. Let $P V_{t}$ denote the set of possible vertices at the end of step $t(0 \leq t \leq K-1)$. Since $v_{\text {end }}$ is the end of step $K$, it is obvious that $P V_{K-1}=N\left(v_{\text {end }}\right)$. So for each $v_{i} \in P V_{K-1}$, we store pathvalue to be $\operatorname{Cost}\left(e_{\text {end,i }}\right)$, and assign $v_{\text {end }}$ to be the successor of such a $v_{i}$. By similar reasoning, we have $P V_{t}=\left\{N\left(v_{i}\right) \mid v_{i} \in P V_{t+1}\right\}$. Now for $t=K-1$ down to 0 , for each $v_{i} \in P V_{t}$, we compare Pathvalue $\left(v_{j}\right)+\operatorname{Cost}\left(e_{j i}\right)$ where $v_{j} \in N\left(v_{i}\right)$, to find the optimal pathvalue for $v_{i}$ and assign its corresponding successor. When the operation is done for $t=0$, starting from $v_{\text {start }}$, we trace back from the successor all the way to $v_{\text {end }}$, which gives us the optimal path.

Note that such an optimal path is only an approximation of the weighted geodesic curve on the continuous surface. However, since our triangulation of the surface is done at the level of voxel size (each cortical surface net has about 500,000 triangles), we have found the discrete path to be a fine enough approximation, as verified by expert inspection. In our implementation, the starting and ending points of a particular sulcus are specified by the user to allow flexibility, which only takes two mouse clicks on a surface rendering. The step number $K$ is usually set to be 300 (large enough for the possible steps for each sulcus) to provide real-time operation. Example automatic traces are shown in Figure 4.

\subsection{Brain Wrapper and Exterior Sulcal Top Curves}

After extracting the interior sulcal bottom curve, we then define the exterior sulcal top. One simple way is to use the signed distance level function $\Psi_{\text {out }}$. While the outer cortical surface $S_{\text {out }}$ is the zero level set of $\Psi_{\text {out }}$, a positive value $\epsilon$ can be chosen so that the $\epsilon$ level set of $\Psi_{\text {out }}$ provides a brain wrapper - 
a surface that wraps around the brain volume, while following indentations at exterior sulcal tops. In our implementation, $\epsilon$ is chosen to be $3 \mathrm{~mm}$, which results in a consistent brain wrapper suitable for subsequent sulcal ribbon extraction. After the brain wrapper surface is extracted, sulcal top curves are automatically traced out on the brain wrapper surface in the same fashion that sulcal bottom curves are on the cortical surface (see Figure 5).

\subsection{Sulcal Ribbon Surface Extraction}

The sulcal ribbon surface corresponds to the medial axis of a particular sulcus. There are different ways of extracting the medial axis of 3D structures, such as those using Voronoi diagrams $[4,13,12]$. The drawbacks of 3D Voronoi methods lie in their algorithmic difficulties caused by sampling problems and pruning procedures. Moreover, a parametric representation of the sulcal ribbon does not follow immediately from these methods.

From the level function $\Psi_{\text {out }}$ and the sulcal bottom and top curves traced out automatically, we have a simple and natural way of defining the entire sulcal ribbon. Our method is based on the fact that the medial axis of a sulcus corresponds to directional local maximum of the signed distance function $\Psi_{\text {out }}$. This is illustrated by the 2D schematic drawing in Figure 1. Figure 1 (b) and (c) show the image and surface view of the signed distance function of the outer cortical boundary in Figure 1(a). The ridge curve in the sulcal region shown in Figure 1(c) is the medial axis of the sulcus, and has the property of being located at a local maximum of the distance function along its normal direction. The 3D case is similar, only differing in that the medial axial ridge curve becomes a surface that has a local maximum of $\Psi_{\text {out }}$ along its normal direction. Our goal now is to capture such a surface through $\Psi_{\text {out }}$.

We will define a sulcal surface mesh $R(u, v)$ on the domain $[0,1] \times[0,1]$, so that parameter $u$ runs in the direction parallel to the sulcal top and bottom curves, while parameter $v$ runs across the sulcal depth. Figure 7 helps in illustrating this process. To start, we map $R(u, 0)$ to be the interior sulcal bottom curve, and $R(u, 1)$ to be the exterior sulcal top curve. We reparameterize $R(u, 1)$ in $u$ so that it has the same $u$ parametric speed as $R(u, 0)$. In this way, we set up a correspondence between the points on the sulcal top and bottom curves, which helps to offer a reasonable concept of sulcal depth discussed later in Section 3.1.

We then realize a piece-wise linear triangulation between the sulcal bottom and top curves to generate the entire mesh as an initialization of the sulcal ribbon surface: $\quad R(u, v)=(1-v) R(u, 0)+v R(u, 1) . \quad$ The number of $v$ iso-parametric curves is chosen to be 20 in our implementation, so that the triangulation of sulcal ribbon surfaces are fine enough to be on the order of a voxel or less. Figure $7(a, b)$ shows such an initialization of a central sulcal surface and a superior frontal sulcal surface.

We then deform the surface according to the following equation while fixing the sulcal bottom curve $R(u, 0)$ : 

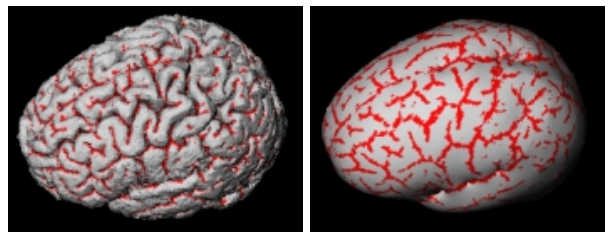

Fig. 3. Left: An outer cortical surface with high maximum principal curvature points shown in red. Right: Flattening makes more high curvature points visible.
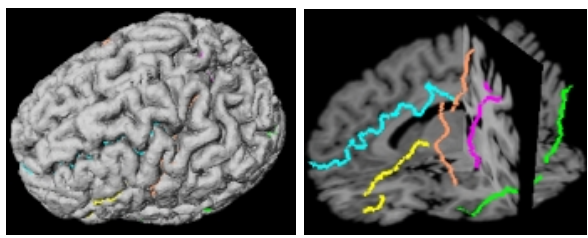

Fig. 4. Automatically traced sulci shown with cortical surface and orthogonal image cards are central (magenta), superior (blue) and inferior (yellow) frontal, superior temporal (green) and pre-central (tan) sulci.
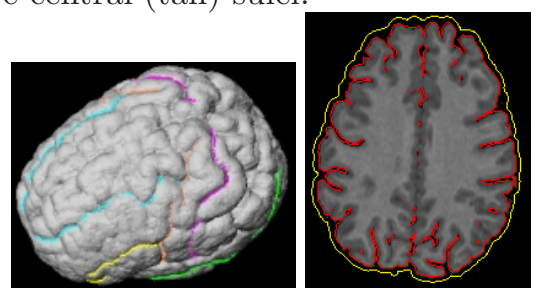

Fig. 5. Left: Corresponding sulcal top curves (also see Figure 4) on brain wrapper. Right: Cut view of brain wrapper (yellow) and cortical surface (red) on axial slice.
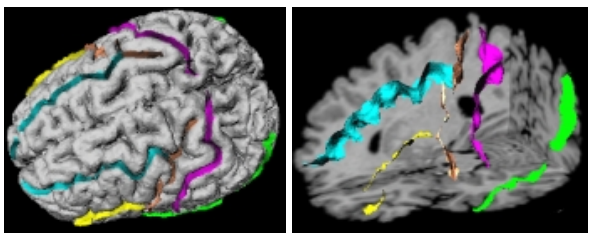

Fig. 6. Sulcal ribbon surfaces corre- Fig. 8. Central (magenta) and supesponding to sulcal curves in Figure 4 rior frontal (blue) sulcal ribbons shown shown on cortical surface and orthogonal image cards.

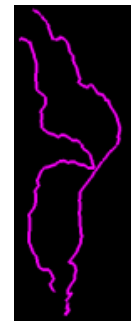

(a1)

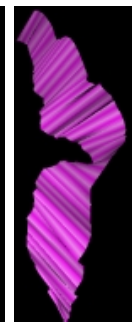

(a2)

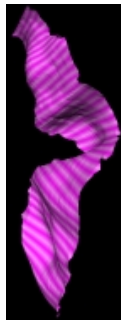

(a3)

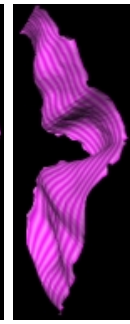

(a4) (b1)

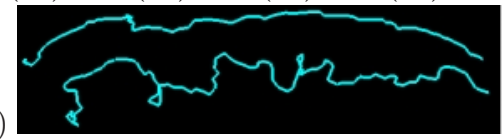

(b2)

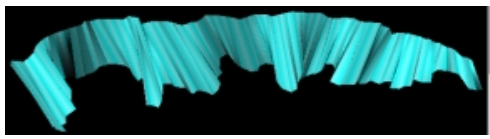

(b3)

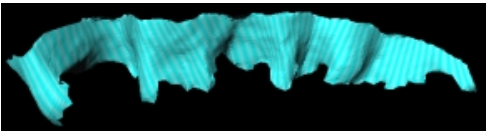

(b4)

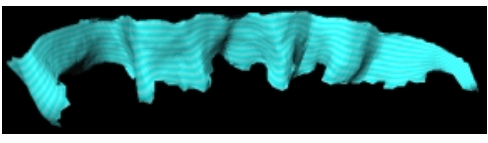

Fig. 7. Deformation of (a): central and (b): superior frontal sulcal ribbons. (1): Sulcal top and bottom curves traced automatically. (2): Piece-wise linear mesh as initialization. Sulcal ribbons with iso-parametric (3): $u$ and (4): $v$ curves superimposed.
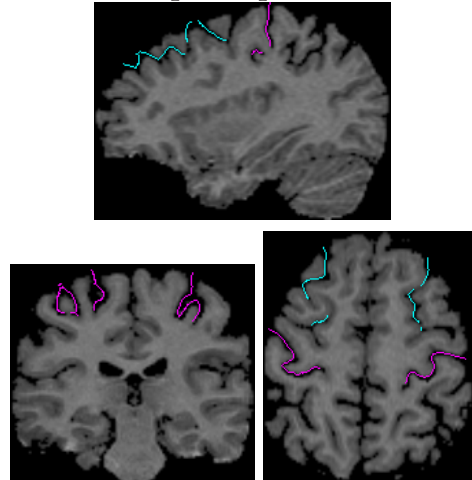

on sagittal, coronal and axial image slices. 


$$
\begin{aligned}
\frac{\partial R(u, v)}{\partial t} & =F_{\text {smooth }}+F_{\text {image }} \\
& =a\left(R_{u u}+R_{v v}\right)+\left(\nabla \Psi_{\text {out }} \cdot N_{R}(u, v)\right) N_{R}(u, v), \quad(u, v) \in[0,1] \times(0,1]
\end{aligned}
$$

where $N_{R}(u, v)$ denotes the unit normal of the sulcal ribbon surface. The first term on the right hand side of the equation guarantees the smoothness of the sulcal ribbon surface, while the second force drives the sulcal surface in its normal direction towards the local maximum of $\Psi_{\text {out }}$ which corresponds to the sulcal medial axis. Thus, the surface deforms to the sulcal medial axis while maintaining a certain smoothness decided by parameter $a$. This equation is discretized on the surface mesh and solved iteratively. The iteration stops when the increase of $\iint_{R(u, v)} \Psi_{\text {out }} d u d v$ falls below a certain threshold, which is set to be 0.05 .

Figure $7(\mathrm{c}, \mathrm{d})$ shows the captured central and superior frontal sulcal surfaces with their iso-parametric $u$ and $v$ curves superimposed. The iso- $u$ parametric curves show the correspondence between the points on sulcal top and bottom curves which is used in the sulcal depth measurement, and the iso- $v$ parametric curves help with the visualization of the convolution across the sulcal depth.

The advantage of our approach is that the information defining the sulcal medial axis is implicit in the signed distance function $\Psi_{\text {out }}$, and by using $\Psi_{\text {out }}$, the problem of finding the sulcal ribbon becomes explicitly one of surface deformation. This formulation avoids the difficulty in tuning the weights of multiple inward force components in the sliding contour model.

\section{Applications}

In this section, we present results of our sulcal ribbon finding algorithm on high resolution MR images (1.5 T GE scanner, SPGR, $1.2 \times 1.2 \times 1.2 \mathrm{~mm}^{3}$ voxel size), and discuss how they can be used for structural and functional analysis of sulci.

We first ran our coupled surfaces algorithm to segment cortical gray matter from white matter and non-brain tissues, which resulted in the outer cortical surface $S_{\text {out }}$ and its level function $\Psi_{\text {out }}$. The segmented cortical gray matter volume was then inspected by an expert, and corrections were made. Accordingly, $S_{\text {out }}$ and $\Psi_{\text {out }}$ were modified locally at the places of correction. This completed the pre-processing step of the sulcal ribbon finding algorithm.

As described in section 2.3, a brain wrapper surface $S_{\text {wrap }}$ was extracted based on $\Psi_{\text {out }}$, and maximum principal curvature was calculated on both $S_{\text {out }}$ and $S_{\text {wrap }}$. Our expert then dropped starting and ending points of sulcal top and bottom curves on $S_{\text {wrap }}$ and $S_{\text {out }}$ respectively. After the sulcal curves were extracted automatically, sulcal ribbon surface was initialized and deformed as described in section 2.4. Software written in $\mathrm{C}++/$ Open Inventor was used for these steps on a SGI Octane machine with a $255 \mathrm{MHz}$ R10000 processor. The automatic tracing of sulcal curves is done in real-time, and the deformation of each ribbon surface takes about $3-5$ seconds. 
Shown in Figure 6 are the ribbon surfaces of central (magenta), superior frontal (blue), inferior frontal (yellow), superior temporal (green) and pre-central (tan) sulci with the outer cortical surface and orthogonal image cards. The cut views of central and superior frontal sulcal ribbon on orthogonal image slices shown in Figure 8 demonstrate the complexity of 3D sulcal convolution captured by our algorithm.
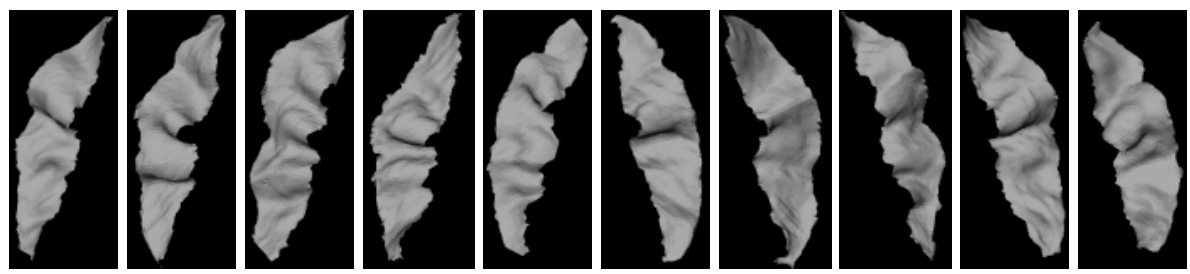

Fig. 9. Corresponding left (left 5) and right (right 5) central sulcal ribbons of 5 normal subjects extracted from MR images using our algorithm.

\subsection{Quantitative Measurement of Central Sulcal Ribbons}

Once the sulcal ribbons are captured in their parametric form, we can make quantitative measurements such as surface area, sulcal depth and sulcal curvature etc. Sulcal ribbon surface area is calculated as the sum of the area of all the triangles used to compose the surface. A reasonable and consistent way to measure sulcal depth is to measure the geodesics to sulcal fundus along the top curve, in other words, the geodesics between $R(u, 1)$ to $R(u, 0)$ for all $u \in[0,1]$. The geodesics are computed using the dynamic programming technique described in Section 2.2, with the cost function set to be simply the length of a step, i.e. $\operatorname{dist}\left(v_{i_{k}}, v_{i_{k+1}}\right)$. Our experiments suggest that most of time the geodesic from $R\left(u_{0}, 1\right)$ to $R\left(u_{0}, 0\right)$ for a particular $u_{0}$ coincides with the iso- $u$ curve $R\left(u_{0}\right)$.

We measured the depth and intrasulcal ribbon surface area of the complete course of the central sulcus in both hemispheres across 15 right handed subjects to demonstrate our methods in an area of interest to neuroscientists. The fundus of the central sulcus is the dividing point for the primary motor region (Brodmann area 4) on the anterior bank and the primary somatosensory strip (Brodmann area 3b) on the posterior bank of the sulcus. In addition to serving as boundaries, the depth of sulci and the total intrasulcal surface area may bear some relationship to the functional capacity of that region. There is a somatotopic mapping of the body on the primary motor and somatosensory regions, such that different regions of these cortical strips process information from different regions of the body. Recent work has suggested that anatomic asymmetry in the depth and surface area of the region of the central sulcus which maps to the hand and arm may be associated with asymmetry in motor function; right 
handers have a deeper central sulcus in this region in the left hemisphere, while the opposite is true in left handers $[18,1,17]$.

Figure 9 shows the central sulcal ribbons of 5 of the subjects to demonstrate the sulcal variability captured by our algorithm. Although not presented here, ongoing work in our lab is comparing regional measurements corresponding to the representation of the hand in this group and a matched comparison group of left handers, in order to test for structure function relationships. Results for the total central sulcus in this group of 15 neurologically healthy young adults of normal general intelligence (IQ mean $\pm S D=108 \pm 15$ ) including 7 males and 8 females between the ages 9 and 41 years are as follows. Average sulcal depth of the complete central sulcal ribbon was $18.12( \pm 1.66) \mathrm{mm}$ on the left and $18.08( \pm 1.67) \mathrm{mm}$ on the right, with no significant right-left difference. Total surface area of the cortical ribbon (corresponding to surface area of one bank, not both sulcal banks) was $1724( \pm 202) \mathrm{mm}^{2}$ on the left and $1764( \pm 205) \mathrm{mm}^{2}$ on the right. These measurements are roughly commensurate with postmortem measurements [17] and prior in vivo morphometry [1], especially considering the differences in measurement procedure (the cited methods were based on interpolation between traces on $2 \mathrm{D}$ slices).

\subsection{Brain Matching With Cortical Constraints}

Another potential use of the extracted sulcal surfaces lies in non-rigid brain warping and cortical atlas building. As distinctive features of the brain, sulcal surfaces can be used as geometric guidelines in shape transformation methodologies. There has already been work toward this direction $[16,6,5]$, and our method offers an alternative to getting a starting point.

\section{Discussion}

We have presented a new approach to automatic 3D sulcal ribbon finding. Dynamic programming is used to automatically extract interior sulcal bottom curves on the outer cortical surface, and exterior sulcal top curves on a brain wrapper computed from the distance function $\Psi_{\text {out }}$ associated with the outer cortical surface. A sulcal ribbon surface is then initialized through a piecewise linear triangulation between the sulcal top and bottom curves, and deformed to sulcal medial axis through the distance function $\Psi_{\text {out }}$. The use of $\Psi_{\text {out }}$ makes the information defining sulcal medial axis implicit, and the resulting surface deformation formulation is simpler without multiple forces to tune. Though a natural follow up to our segmentation method, our sulcal ribbon finding algorithm can be adapted to follow other segmentation procedures. By allowing the user to define a sulcal ribbon with a few mouse clicks, our method offers automation, flexibility and real-time operation.

All the sulcal ribbon surfaces captured by our algorithm are evaluated slice by slice on axial image slices by an expert. The positions of the ribbon surfaces are always within one voxel's distance from the sulcal medial axis by visual 
inspection. However, since any type of expert tracing of the sulcal ribbon suffers from its limitation in capturing the $3 \mathrm{D}$ nature of sulci, we feel the best way to do full quantitative analysis of our algorithm is to create a phantom with known convolutions, and experiment on its images.

Our method for extracting the brain wrapper has potential for further improvement. There are no well defined methods to locate the exterior top edge of a sulcus. Although our way of extracting a fixed $\epsilon$ level set surface is consistent in it own right, ideally different values of $\epsilon$ need to be chosen for different sulci in order to obtain a more geometry-specific definition. Other directions of future research include localized sulcal measurement and shape analysis, and the study of structure and function relationships in the sulcal region.

\section{Acknowledgements}

This work was supported in part by NIH grant NINDS R01 NS35193, NSF grant IRI-9530768, and NIH grant NICHD 5 P01 HDIDC 35482.

\section{References}

1. K. Amunts et al. Asymmetry in the human motor cortex and handedness. Neuroimage, 4(3 Pt 1):216-22, Dec 1996. 156

2. D.H. Ballard and C.M. Brown. Computer Vision, chapter 4. Prentice-Hall Inc., New Jersey, 1982. 151

3. R. Bellman and R. Kalaba. Dynamic Programming and Modern Control Theory. Academic Press Inc, 1965. 151

4. H. Blum. Models for the Perception of Speech and Visual Form. MIT Press, 1967. 152

5. H. Chui, J. Rambo, R. Schultz, J. Duncan, and A. Rangarajan. Registration of cortical anatomical structures via robust 3D point matching. In Proc. IPMI, 1999. 156

6. D.L. Collins, G.L. Goualher, and A.C. Evans. Non-linear cerebral registration with sulcal constraints. In Proc. MICCAI, pp.974-984, MIT, 1998. 156

7. S. Gibson. Constrained elastic surface nets: Generating smooth surfaces from binary segmented data. In Proc. MICCAI, pp.888-898, MIT, 1998. 150

8. G.L. Goualher, C. Barillot, and Y.Bizais. Modeling cortical sulci using active ribbons. Int. Journal of PRAI, 11(8):1295-1315, 1997. 148

9. G.L. Goualher, D.L. Collins, C. Barillot, and A.C. Evans. Automatic identification of cortical sulci using a 3D probabilistic atlas. In Proc. MICCAI, pp.509-518, 1998. 148

10. N. Khaneja, M.I. Miller, and U. Grenander. Dynamic programming generation of curves on brain surfaces. IEEE Trans. PAMI, 20(11):1260-1265, 1998. 151

11. J. Mangin, J. Regis, I. Bloch, V. Frouin, Y. Samson, and J. LopezKrahe. A MRF based random graph modeling the human cortical topography. In Proc. CVRMed, pp.177-183, Nice, 1995. 148

12. L.R. Nackman and S.M. Pizer. Three-Dimensional shape description using the symmetric axis transformation I: Theory. IEEE Trans. PAMI, 7(2):187-202, 1985. 152 
13. M. Naf, O. Kubler, R. Kikinis, M.E. Shenton, and G. Szekely. Characterization and recognition of $3 \mathrm{~d}$ organ shape in medical image analysis using skeletonization. In Proc. MMBIA, pp.139-150, 1996. 152

14. J. Rademacher, V.S. Caviness Jr, H. Steinmetz, and A.M. Galaburda. Topographical variation of the human primary cortices: implications for neuroimaging, brain mapping, and neurobiology.. Cerebral Cortex, 3(4):313-329, 1993. 148

15. M. Vaillant and C. Davatzikos. Finding parametric representations of cortical sulci using an active contour model. Medical Image Analysis, pp.295-315, Sep 1997. 148

16. M. Vaillant and C. Davatzikos. Mapping the cerebral sulci: Application to morphological analysis of cortex and to non-rigid registration. In Proc. IPMI, pp.141-154, 1997. 156

17. L.E. White, T.J. Andrews, C. Hulette, A. Richards, M. Groelle, J. Paydarfar, and D. Purves. Structure of the human sensorimotor system. II: Lateral symmetry. Cerebral Cortex, 7(1):31-47, 1997. 156

18. L.E. White, G. Lucas, A. Richards, and D. Purves. Cerebral asymmetry and handedness. Nature, 368(6468):197-8, 1994. 156

19. X. Zeng, L.H. Staib, R.T. Schultz, and J.S. Duncan. Segmentation and measurement of the cortex from 3D MR images. In Proc. MICCAI, pp.519-530, 1998. 149, 150

20. X. Zeng, L.H. Staib, R.T. Schultz, and J.S. Duncan. Volumetric layer segmentation using coupled surfaces propagation. In Proc. CVPR, pp.708-715, 1998. 150 